\title{
Functional analysis of the acetylation of human p53 in DNA damage responses
}

\author{
Sun-Ku Chung ${ }^{2}$, Shengyun $\mathrm{Zhu}^{1}$, Yang $\mathrm{Xu}^{2}$, Xuemei $\mathrm{Fu}^{1,3 凶}$ \\ ${ }^{1}$ Shenzhen Children's Hospital, 7019 Yitian Road, Shenzhen, Guangdong 518026, China \\ 2 Division of Biological Sciences, University of California, San Diego, 9500 Gilman Drive, La Jolla, CA 92093, USA \\ ${ }^{3}$ Chongqing Medical University, Chongqing 400016, China \\ $\triangle$ Correspondence: fxmzj2004@163.com (X. Fu) \\ Received February 9, 2014 Accepted February 24, 2014
}

\begin{abstract}
As a critical tumor suppressor, p53 is inactivated in human cancer cells by somatic gene mutation or disruption of pathways required for its activation. Therefore, it is critical to elucidate the mechanism underlying p53 activation after genotoxic and cellular stresses. Accumulating evidence has indicated the importance of posttranslational modifications such as acetylation in regulating p53 stability and activity. However, the physiological roles of the eight identified acetylation events in regulating $\mathrm{p} 53$ responses remain to be fully understood. By employing homologous recombination, we introduced various combinations of missense mutations (lysine to arginine) into eight acetylation sites of the endogenous p53 gene in human embryonic stem cells (hESCs). By determining the p53 responses to DNA damage in the p53 knock-in mutant hESCs and their derivatives, we demonstrate physiological importance of the acetylation events within the core domain (K120 and K164) and at the C-terminus (K370/372/373/381/382/ 386 ) in regulating human $\mathrm{p} 53$ responses to DNA damage.
\end{abstract}

KEYWORDS human embryonic stem cells (hESCs), p53, acetylation, homologous recombination,

DNA damage, cancer

\section{INTRODUCTION}

Tumor suppressor p53 is a transcription factor that directly activates the transcription of hundreds of genes, including p21, Mdm-2, noxa, and puma that are mediating p53-

Sun-Ku Chung and Shengyun Zhu contributed equally to this work. dependent functions (Vousden and Prives, 2009). In addition, p53 also directly suppresses the expression of a number of genes, such as Nanog (Lin et al., 2005). While p53 remains in an unstable and inactive state in normal cells in the absence of exogenous stresses, its protein level and transcription activity are significantly induced in response to DNA damage and other cellular stresses (Vousden and Prives, 2009). The interaction between p53 and E3 ligases such as MDM2, all transcription targets of p53, leads to the ubiquitination and rapid degradation of $p 53$. In addition, the interaction between $\mathrm{p} 53$ and $\mathrm{Mdm} 2$ or $\mathrm{MdmX}$ suppresses the transcriptional activities of $p 53$. These negative regulatory pathways are critical to prevent the hyperactivation of p53 that can have devastating effects on organismal survival and aging (Campisi, 2005; Liu et al., 2010).

p53 is critical to suppress cancer development in humans, and the loss of wild-type p53 activity through direct somatic gene mutation or disruption of pathways important for p53 activation is required for cancer progression (Vousden and Prives, 2009). Therefore, it is critical to elucidate the pathways that activate p53. Accumulating evidence indicates that posttranslational modifications of $\mathrm{p} 53$, including phosphorylation and acetylation, are involved in regulating $\mathrm{p} 53$ stability and activity. While phosphorylation of p53 at $\mathrm{N}$-terminus is important to activate some of p53dependent functions, none of the phosphorylation events are critical for p53 activation (Chao et al., 2003; Chao et al., 2006). Recent studies indicate that human p53 can be acetylated at multiple lysine residues by Tip60 and CBP, including K120, K164, K320, and the C-terminal K370/372/ 373/381/382/386 (Brooks and Gu, 2011). Using human cancer cell lines expressing p53 with various lysine to arginine mutations, these studies indicate that K120R, $\mathrm{K} 164 \mathrm{R}$ as well as K120/164R double mutations partially impaired the p53-dependent functions (Sykes et al., 2006; 
Tang et al., 2006; Tang et al., 2008). In addition, mutation of the eight acetylation sites to arginine abolishes the p53dependent functions, suggesting that these acetylation events play synergistic and critical roles in activating p53 (Sykes et al., 2006; Tang et al., 2006, 2008).

The roles of acetylation have also been examined by knock-in studies in mouse and ESCs. Employing p53 K3R knock-in mouse, Li and colleagues demonstrate that acetylation of mouse p53 at K117/161/162 (corresponding to human $\mathrm{K} 120 / 164$ ) is required for activating p53-dependent transcription but dispensable for p53 stabilization ( $\mathrm{Li}$ et al., 2012). By generating p53 K120/164R knock-in hESCs, we demonstrate that these two acetylation events are required for p53-dependent transcription and stabilization (Zhang et al., 2014). These findings demonstrate that acetylation might have distinct impact on p53 stability and activity in mouse and human cells.

\section{RESULTS}

\section{Generation of K6R and K8R knock-in hESCs}

To determine the functional interaction of the acetylation events within the core domain and the C-terminus of p53, we employed the same genetic approach to reveal the physiological importance of eight acetylation events in regulating human $\mathrm{p} 53$. The strategy to introduce the $\mathrm{K}$ to $\mathrm{R}$ mutations (K370/372/373/381/382/386R, K6R; K120/164/370/372/373/ $381 / 382 / 386 R$, K8R) into the endogenous p53 gene of hESCs is the same as we described for the K120/164R (K2R) knock-in mutation (Zhang et al., 2014) and is described in Fig. 1. We introduced K8R mutations into two independent hESC lines (HUES8 and HUES9) and K6R mutations into the HUES8 hESCs. These knock-in hESCs have normal karyotypes and can form well-differentiated teratomas in immunodeficient mice, confirming their pluripotency (Fig. 2A and 2B). The full length cDNA of the p53 gene in the knock-in hESCs was sequenced to confirm that only the $\mathrm{K}$ to $\mathrm{R}$ mutations but no other mutations were introduced into the p53 knock-in allele.

p53 stabilization is abolished in K8R hESCs and fibroblasts after DNA damage

Our previous studies have shown that K120/164R (K2R) mutation is important for p53 stabilization and activation in hESCs after DNA damage (Zhang et al., 2014). Cell lines studies indicate that the acetylation at K120/164 works synergistically with acetylation at the C-terminus (Tang et al., 2008). To determine the physiological roles of the acetylation of p53, we examined the impact of K8R mutations on the p53 stability and activity in hESCs after DNA damage. Consistent data were obtained from both K8R knock-in hESC lines (HUES8 and HUES9), and the representative data are presented.
To determine the impact of K8R on p53 stability, the protein levels of p53 in $\mathrm{p} 53^{+/-}, \mathrm{K} 8 \mathrm{R}$ and $\mathrm{p} 53^{-/-} \mathrm{hESCs}$ at different time points after DNA damage. While the protein levels of $\mathrm{p} 53$ were significantly increased in ${\mathrm{p} 53^{+/-}} \mathrm{hESCs}$ after DNA damage, they remained unchanged in K8R hESCs after DNA damage, indicating that DNA damage induced stabilization of $\mathrm{p} 53$ is abolished in K8R hESCs (Fig. 3A). Consistent with the findings that p53 is constitutively acetylated (Tang et al., 2008), the basal levels of p53 protein were significantly lower in K8R hESCs than in $\mathrm{p} 53^{+/-}$ hESCs, indicating that acetylation stabilizes p53 in the absence of exogenous stresses.

To confirm that the role of this acetylation in stabilizing p53 is not cell-type specific, we derived the fibroblasts from $\mathrm{p} 53^{+/-}$and K8R hESCs, and determined the p53 protein levels in these cells after DNA damage. Our data confirmed that the basal levels of p53 protein were much lower in K8R fibroblasts than those in $\mathrm{p} 53^{+/-}$fibroblasts and could not be induced by DNA damage (Fig. 3B). Therefore, we concluded that acetylation is required to stabilize $p 53$. In further support of this conclusion, p53 was hyper-ubiquitinated in K8R hESCs and fibroblasts

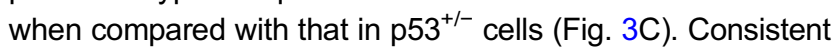
with the increased ubiquitination of p53 in K8R cells, the interaction between Mdm2 and p53 was significantly increased in K8R hESCs than in $\mathrm{p} 53^{+/-}$hESCs when analyzed by coimmunoprecipitation (Fig. 3D). In addition, treatment with Nutlin-3, which specifically disrupts the interaction between Mdm2 and p53, significantly increases the protein levels of p53 in K8R hESCs, supporting the notion that the decreased protein levels of p53 in K8R hESCs are primarily due to the increased p53Mdm2 interaction (Fig. 3E). In summary, these findings support the notion that acetylation is required for p53 stabilization by promoting p53-Mdm2 interaction.

\section{p53-dependent functions are abolished in K8R hESCs and fibroblasts}

Previous findings have shown that hESCs undergo p53dependent apoptosis and cell cycle $\mathrm{G}_{2} / \mathrm{M}$ checkpoint after DNA damage (Song et al., 2010). Therefore, these p53dependent functions were analyzed in K8R hESCs after DNA damage. p53-dependent apoptosis was abolished in the K8R hESCs after DNA damage induced by doxorubicin (Fig. 4A). In addition, p53-dependent $\mathrm{G}_{2} / \mathrm{M}$ checkpoint was essentially compromised in K8R hESCs after IR (Fig. 4B). Consistent with these findings, p53-dependent transcription of target genes, including p21, Mdm2, and Puma, was abolished in K8R hESCs after IR (Fig. 4C). To confirm that the impact of K8R mutation on p53 transcription activity is not cell type dependent, we analyzed the p53-dependent induction of p21 in the fibroblasts derived from K8R hESCs, indicating that p53dependent p21 expression is abolished in K8R fibroblasts both before and after DNA damage (Fig. 4D). Therefore, we concluded that p53-dependent functions are abolished in K8R hESCs after DNA damage. 
A

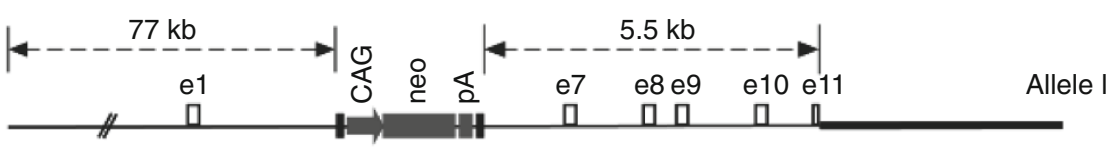

B

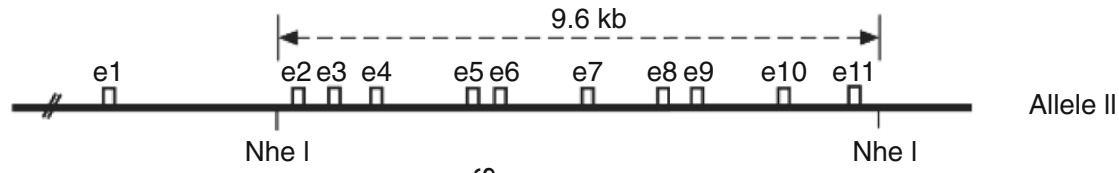

C



D

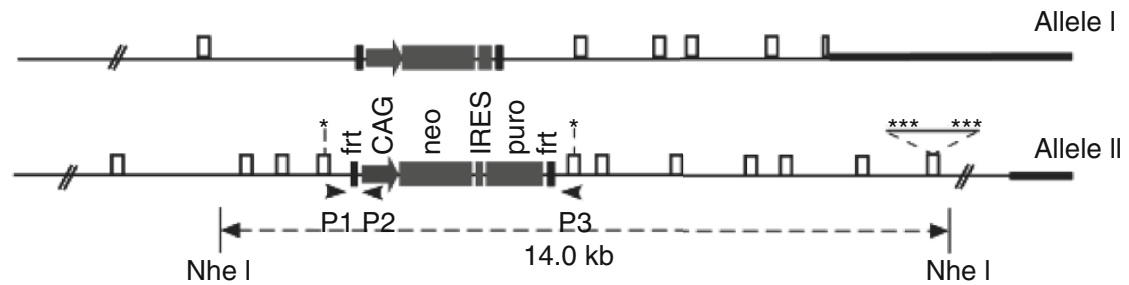

E

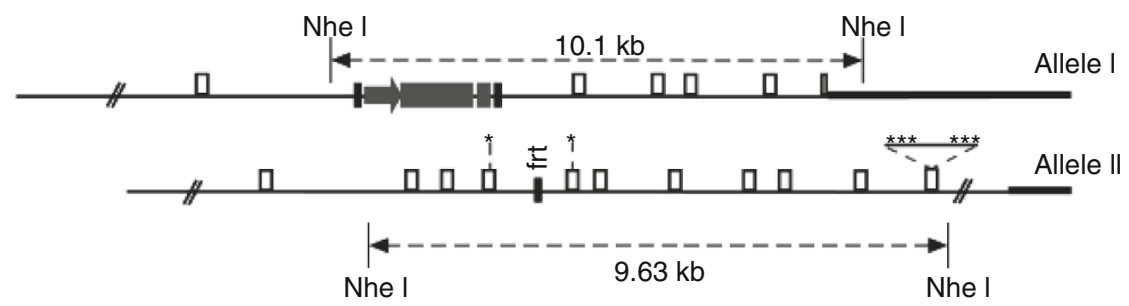

F

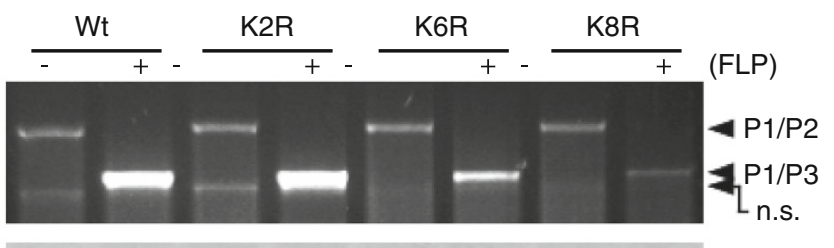

G

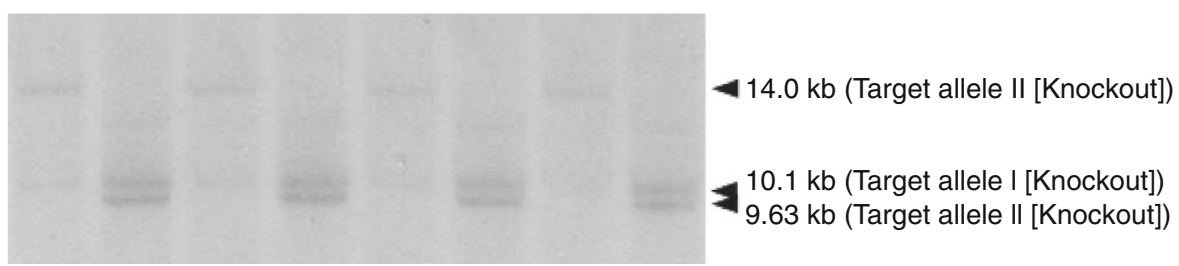

Figure 1. Generation of p53 acetylation site knock-in hESCs. (A) Genome configuration of the p53 knockout allele in parental p53 $3^{+/-}$hESCs. (B) Germline configuration of p53 WT allele in p53 ${ }^{+/-}$hESCs. (C) The BAC-based knock-in vector to introduce K to R mutations into the endogenous p53 gene in hESCs. The eight $K$ to R mutations are indicated by asterisks. (D) Homologous recombination between the WT p53 allele of $\mathrm{p} 53^{+/-} \mathrm{hESCs}$ and the targeting vector led to knock-in hESCs with the selection cassette inserted in the targeted allele. The PCR primers to screen for the FLP/FRT-mediated deletion are indicated. (E) Knock-in hESCs. Transient expression of FLP in the targeted hESCs led to the excision of the selection marker from the knock-in allele. The sizes of the Nhel restriction fragments are indicated. (F) PCR (top panel) and Southern blotting (bottom panel) analyses of the knock-in hESCs. Deletion of the selection cassette led to the loss of PCR amplification by primers P1/P2 and the gain of PCR amplification by primers P1/P3. Genomic DNA was digested by Nhe1 for Southern blotting. 
A

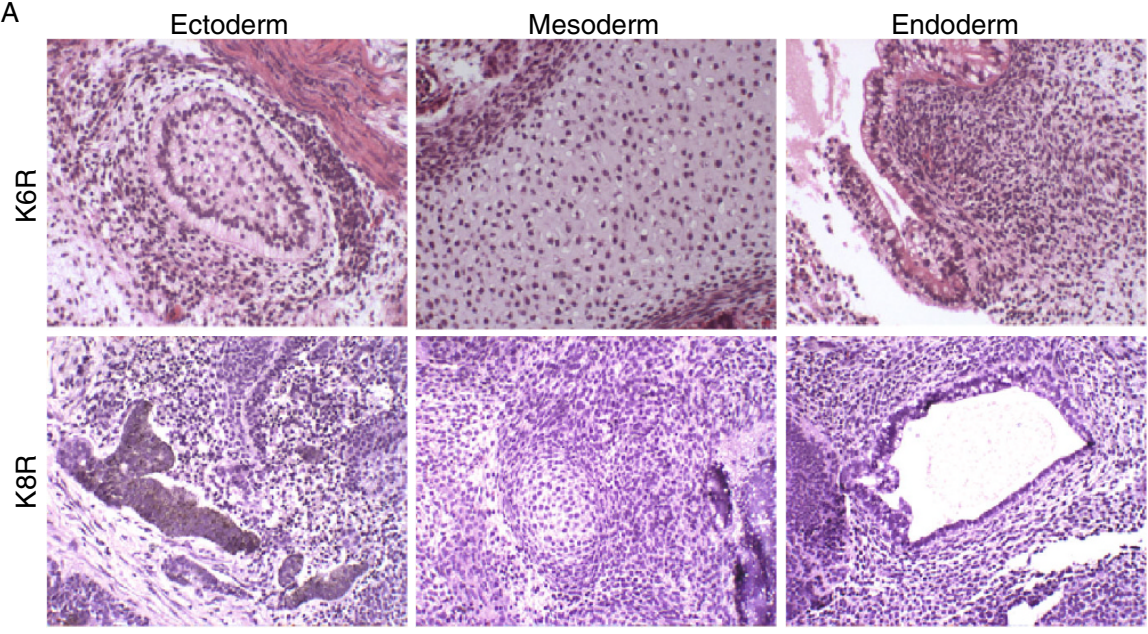

B


Figure 2. Characterization of $p 53 \mathrm{~K} 6 \mathrm{R}$ and K8R knock-in hESCs. (A) Metaphase cells of the knock-in hESCs were examined and exhibited normal karyotypes. Representative images are shown. (B) Knock-in hESCs formed well-differentiated teratomas in SCID mice. Cells of each of the three germ layers were identified in the teratomas.

Functional dissection of acetylation within the core domain and $\mathrm{C}$-terminus

To dissect the contribution of the acetylation events within the core domain and C-terminus to activate p53 responses, we examined the p53 responses to DNA damage in K6R knock-in hESCs and compared to those in K2R (K120/164R) hESCs. In contrast to the findings that p53 protein levels in K8R hESCs were much lower than those in $\mathrm{p}^{53^{+/-}} \mathrm{hESCs}$ and could not be increased by DNA damage, the p53 protein levels in K6R hESCs were induced by DNA damage (Fig. 5A). While the half-life of p53 in K2R hESCs is significantly reduced when compared to that in ${\mathrm{p} 53^{+/-}} \mathrm{hESCs}$ (Zhang et al., 2014), the half-life of p53 in p53 ${ }^{+/-}$hESCs is similar to that in K6R hESCs (Fig. 5B). In contrast to that of $\mathrm{K} 8 \mathrm{R}$, the interaction between p53 and Mdm2 is only modestly increased in K6R hESCs, indicating that acetylation within the core domain plays a key role in disrupting the interaction between p53 and Mdm2 (Fig. 5C). However, the ubiquitination levels were modestly reduced in K6R hESCs, supporting the notion that the C-terminal lysine residues are targeted for ubiquitination (Fig. 5D).

\section{The p53-dependent functions in K6R hESCs} and fibroblasts

In contrast to that in K8R and K2R hESCs that are abolished in p53-dependent gene expression after DNA damage (Fig. 6A), p53-dependent gene expression was higher in K6R hESCs than those in ${\mathrm{p} 53^{+/-}}$control hESCs both before and after DNA damage, indicating that $\mathrm{K} 6 \mathrm{R}$ mutation increases the p53 responses to DNA damage in hESCs (Fig. 6A). In further support of this conclusion, p53-dependent apoptosis was abolished in K2R hESCs but increased in K6R hESCs after IR (Fig. 6B). Therefore, in contrast to the cell line data suggesting that $\mathrm{K} 2 \mathrm{R}$ and $\mathrm{K} 6 \mathrm{R}$ mutations synergistically disrupt p53-dependent transcription but not p53 stability (Li et al., 2012), our findings indicate that K2R and K6R mutations have opposite impacts on p53 stability and activity.

\section{DISCUSSION}

By generating acetylation site mutant knock-in hESCs, we revealed the physiological roles of acetylation events within 
A

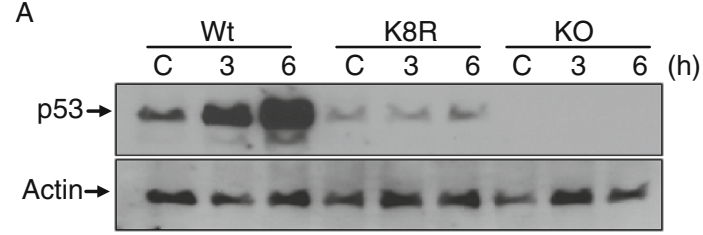

B

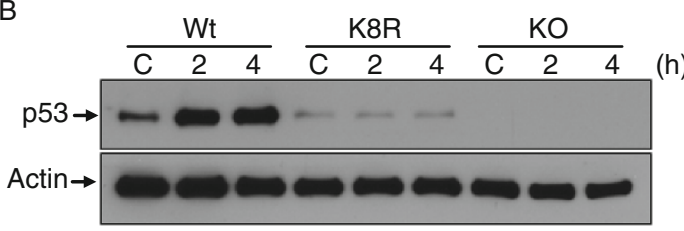

D

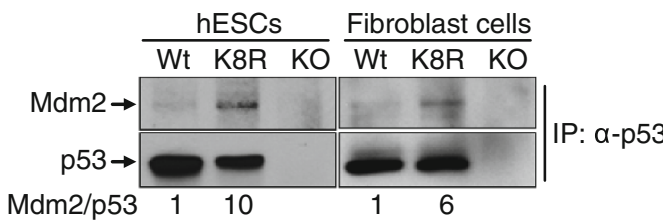

C

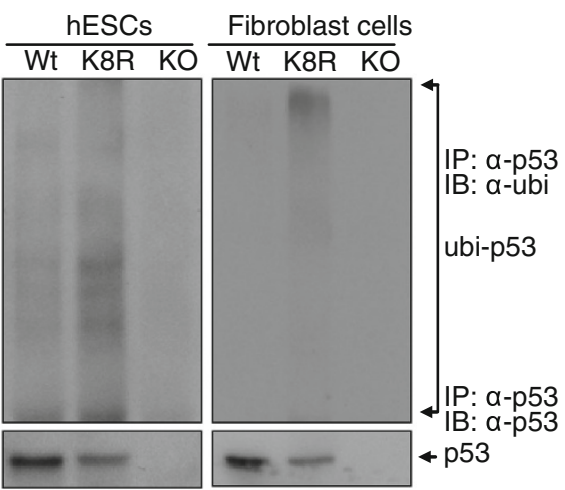

E

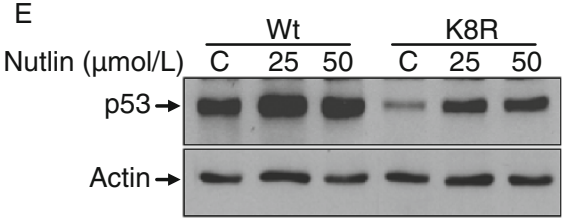

Figure 3. $\mathrm{p} 53$ stabilization is compromised in K8R hESCs and fibroblasts. The protein levels of p53 in p53 ${ }^{+/-}, \mathrm{K}^{2} \mathrm{R}$ and $\mathrm{p} 53^{-/-}$ hESCs (A) and fibroblasts (B) at different time points after DNA damage induced by doxorubicin. The genotypes and the hours of doxorubicin treatment are indicated. The basal levels of p53 in K8R cells are lower than those of p53 ${ }^{+/}$cells. (C) The ubiquitination of p53 in p53 ${ }^{+/-}$and K8R hESCs and fibroblasts. p53 ${ }^{-/-}$hESCs and fibroblasts were used as negative controls for specificity of immunoprecipitation. (D) The interaction between p53 and Mdm2 in p53 ${ }^{+/-}$and K8R cells by co-immunoprecipitation. p53 was

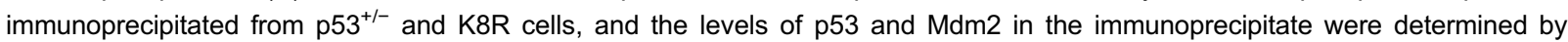
Western blotting. (E) The stabilization of $\mathrm{p} 53$ in $\mathrm{p} 53^{+/-}$and K8R hESCs by treating with Nutlin-3 for $24 \mathrm{~h}$. The concentrations of Nutlin3 used are indicated on the top.

the core domain and C-terminus and their functional interaction in activating human p53. There are several distinctions between our conclusion and those reached in previous cell line and mouse studies (Tang et al., 2008; Li et al., 2012). First, while previous findings indicate that acetylation is required for p53-dependent activity but dispensable for p53 stabilization, our findings indicate that acetylation is required for p53 stabilization. Second, previous findings suggest that the acetylation at the core domain plays synergistic roles with the acetylation at the C-terminus in activating p53-dependent transcription. However, our findings indicate that the mutations of the acetylation sites within the core domain and at the C-terminus have opposite impact on p53 stability and activity. These discrepancies could be due to the intrinsic difference in the sequence of the mouse and human p53 gene. In addition, the overexpression of various p53 mutants in human cancer cell lines could also mask the important roles of acetylation in p53 stabilization.

Our findings indicate that acetylation within the core domain but not at the C-terminus plays a key role in the disruption of the interaction between p53 and its E3 ligase Mdm2. In this context, in addition to the N-terminus of p53, $\mathrm{Mdm} 2$ binds to other regions of $\mathrm{p53}$, including the core domain (Vousden and Prives, 2009). Despite the finding that p53-Mdm2 interaction is modestly increased in K6R cells, p53 is more stable in K6R cells with reduced ubiquitination when compared to wild type p53, indicating that the C-terminal lysine residues are the preferential targets for ubiquitination. In addition, the increased stability of p53 in K6R cells after DNA damage leads to higher p53-dependent transcription and apoptosis after DNA damage. Together with previous findings, our data demonstrate the critical and complex roles of acetylation in activating human p53 response to DNA damage and could provide the foundation to develop novel strategies to activate p53 in human cancer cells harboring WT but dysfunctional p53.

\section{MATERIALS AND METHODS}

Generation of acetyl defective p53 mutants

RP11-199F11, BAC clone including human p53, was purchased from Invitrogen. The BAC based targeting vector was generated and modified by recombineering in the $E$. coli strain SW102. To obtain ${\mathrm{p} 53^{+/-}}^{+}$hESCs, Neomycin marker was firstly used for selection (Song et al., 2010). Each of wild type, or mutant p53 BAC vector commonly had selection cassette (CAG-Neo-IRES-PuropolyA) through insertion into intron 4 of $p 53$. By targeting each

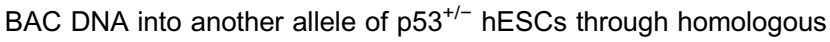
recombination, each clone harboring wild type, or acetyl mutant of

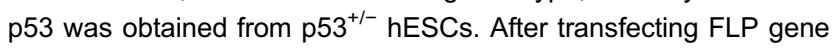
into each clone, cDNA sequence of each clone was confirmed to mutation sites. 


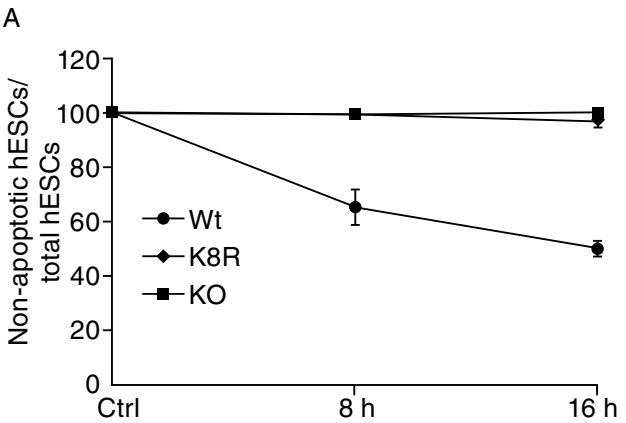

C

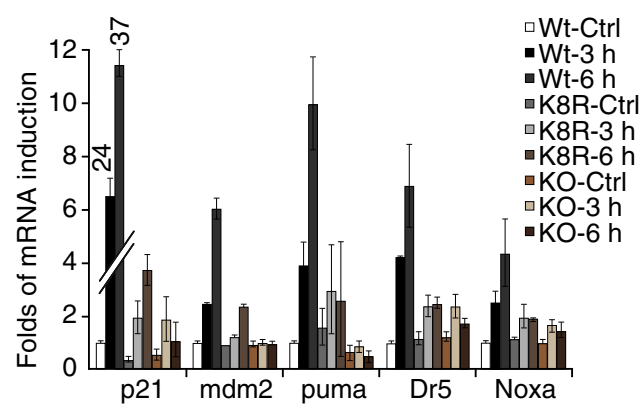

B

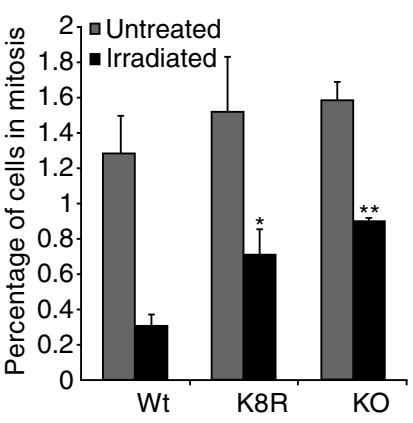

D

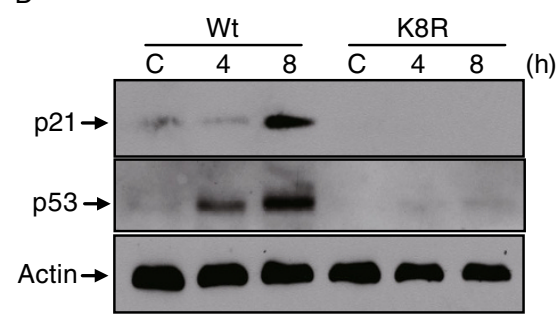

Figure 4. p53-dependent functions are abolished in K8R hESCs and functions. (A) p53-dependent apoptosis in $p 53^{+/-}, K_{8 R}$ and p53 ${ }^{-/-}$hESCs after 5 Gy of IR. The apoptotic cells were identified by staining with Annexin V. (B) The p53-dependent $\mathrm{G}_{2} / \mathrm{M}^{-}$

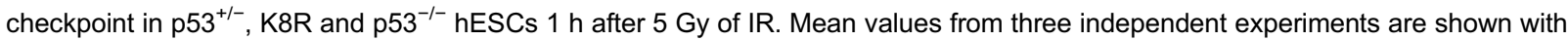
SD. The $P$ value between K8R cells and $p 53^{+/-}$cells is $0.02\left(^{*}\right)$, while the $P$ value between $p 53^{-/-}$cells and $p 53^{+/-}$cells is $0.001\left(^{* *}\right)$ as shown is Fig. 4B. (C) p53-dependent gene expression in $\mathrm{p} 53^{+/-}, \mathrm{K} 8 \mathrm{R}$ and $\mathrm{p} 53^{-1-} \mathrm{hESCs}$ after doxorubicin treatment. The mRNA levels of p53 target genes were determined by quantitative real time PCR and standardized by the mRNA levels of GAPDH. (D) p53dependent induction of $\mathrm{p} 21$ is abolished in the fibroblasts derived from K8R hESCs after doxorubicin treatment. The time points after the treatment are indicated on the top.

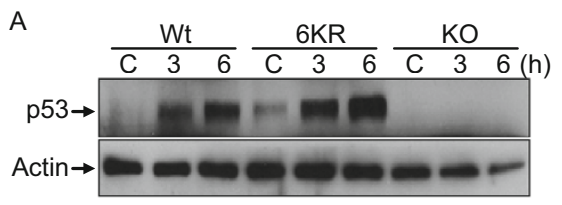

B

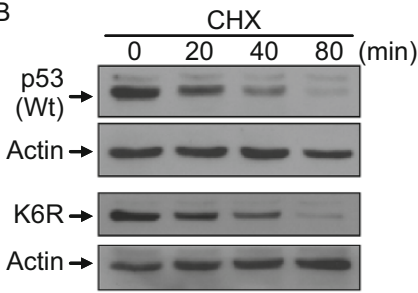

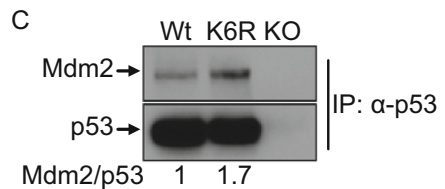

D

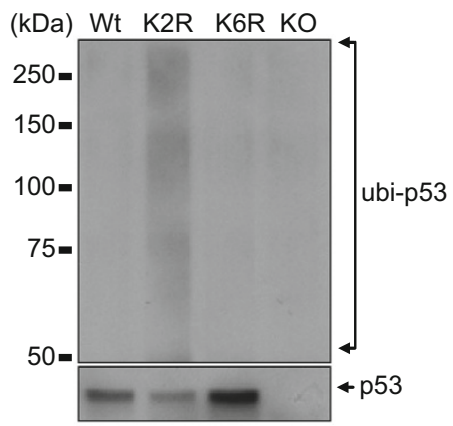

Figure 5. The stability of p53 protein in K6R knock-in hESCs. (A) The protein levels of p53 in p53 $3^{+/-}$and $\mathrm{K} 6 \mathrm{R}$ hESCs after doxorubicin treatment. The p53 and actin are indicated. (B) The protein levels of p53 in p53 ${ }^{+/-}$and K6R hESCs at different time points after treatment with cycloheximide (CHX) that inhibits protein synthesis. (C) The interaction between p53 and Mdm2 in p53 ${ }^{+/-}$and K6R hESCs was analyzed by co-immunoprecipitation. $p 53^{-1-} \mathrm{hESC}$ sere used as a negative control. (D) The ubiquitination of $\mathrm{p} 53$ in $\mathrm{p} 53^{+/-}, \mathrm{K} 2 \mathrm{R}$ and K6R hESCs. The p53 protein was immunoprecipitated and the amount of ubiquitination was analyzed by antiubiquitin antibody. $\mathrm{p} 53^{-1-} \mathrm{hESCs}$ were used as a negative control. 

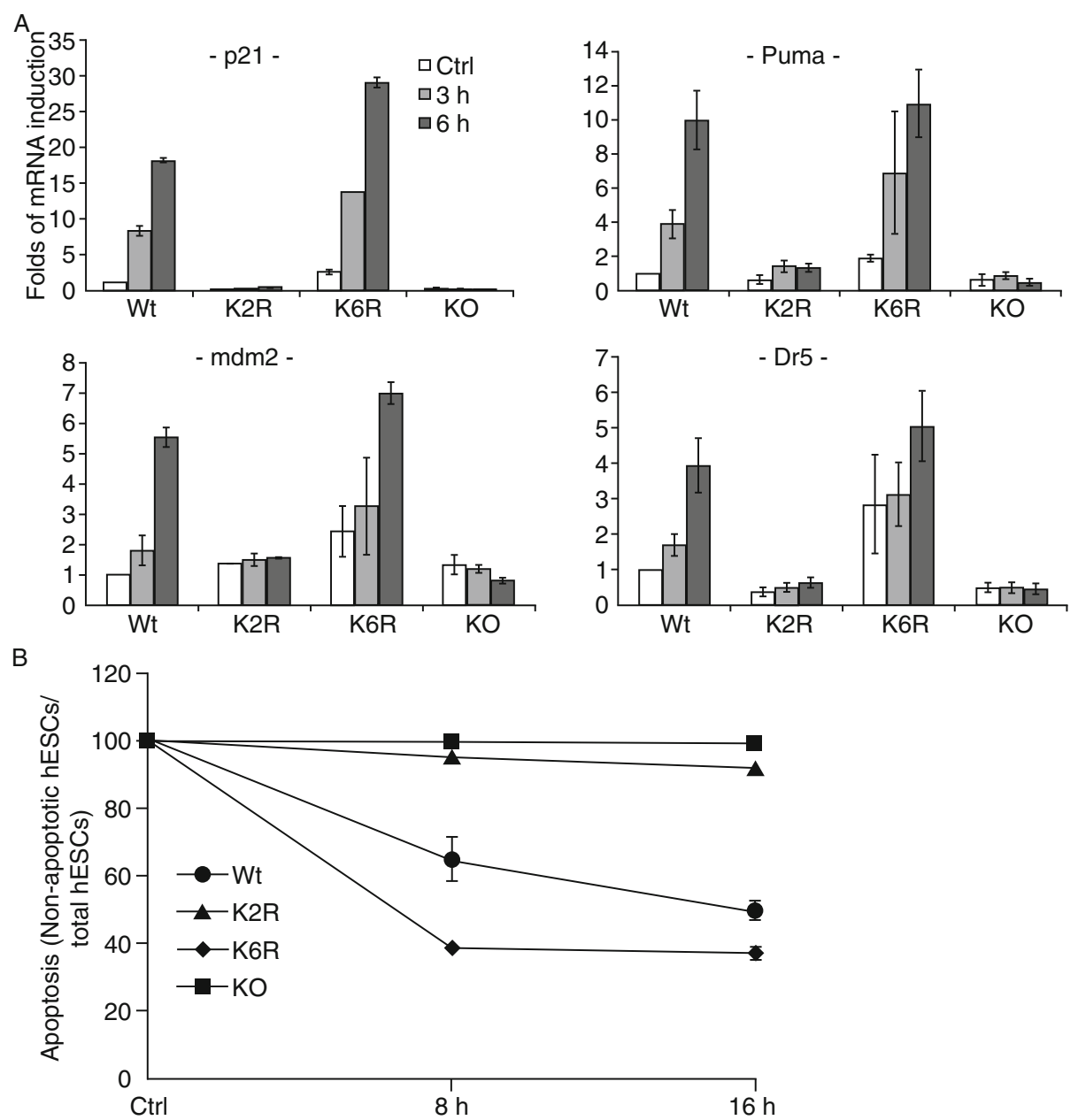

Figure 6. p53-dependent functions are abolished in K2R cells but increased in K6R cells. (A) p53-dependent gene expression

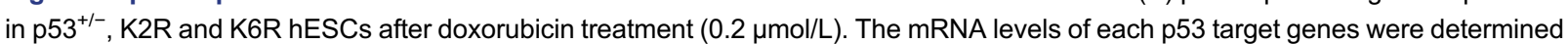
by quantitative PCR and standardized with the mRNA levels of GAPDH. (B) p53-dependent apoptosis in p53 ${ }^{+/-}$, K2R and K6R hESCs after doxorubicin treatment. The apoptotic cells were identified by staining with Annexin V. Mean value from three independent experiments are shown with SD. $n=3$.

Cell culture for hESCs

In the presence of feeder layer, the HUES cells were cultured on feeder layer in knockout DMEM supplemented with 10\% knockout serum replacement (KSR), $10 \%$ plasmanate, $1 \%$ PenStrep, $1 \%$ Glutamine, $1 \%$ nonessential amino acids, $10 \mathrm{ng} / \mathrm{mL}$ bFGF, and $55 \mu \mathrm{mol} / \mathrm{L} \beta$-mercaptoethanol as described (Cowan et al., 2004). In the absence of feeder layer, HUES cells were cultured on matrigelcoated plates in the mTeSR1 medium with $5 \times$ supplement. All tissue culture reagents were purchased from Invitrogen.

\section{Western blotting and immunoprecipitation analysis}

Protein extracts from $\mathrm{hESC}$ s or fibroblasts derived from teratoma were loaded on $6 \%-10 \%$ SDS-PAGE gel and transferred to nitrocellulose membrane, which was probed with a monoclonal antibody against $\mathrm{p} 53$ (pAb1801; Santa Cruz Biotechnology, Santa Cruz, CA), monoclonal antibody against Mdm2 (Ab-2; Oncogene Research), polyclonal antibody against p21 or $\beta$-actin (Santa Cruz Biotechnology). The membrane was subsequently probed with a horseradish peroxidaseconjugated secondary antibody and developed with ECL PLUS (Amersham, Piscataway, NJ). For co-immunoprecipitation analysis, 1-2 mg of whole cell protein extracts was immunoprecipitated with anti-p53 antibody (FL393; Santa Cruz Biotechnology). The amount of p53 and Mdm2 in the immunoprecipitate was analyzed by Western blotting using monoclonal antibody against p53 (pAb1801; Santa Cruz Biotechnology, Santa Cruz, CA), monoclonal antibody against Mdm2 (SMP-14; Santa Cruz Biotechnology, Santa Cruz, CA).

\section{Quantitative real-time PCR}

Real-time PCR was performed as previously described (Song et al., 2010). The sequence of the primers was as follows: p21, $5^{\prime}-A C C$ TGGAGACTCTCAGGGTCG-3' for forward primer, 5'-TTAGGGCT TCCTCTTGGAGAAGAT-3' for reverse primer; Mdm2, 5'-ATCGGA CTCAGGTACATCTGTGAG-3' for forward primer, 5'-AGGTTTCTC 
TTCCTGAAGCTCTTG-3' for reverse primer; Puma, 5'-GACGACCT CAACGCACAG-3' for forward primer, 5'-CTAATTGGGCTCCATC TCG-3' for reverse primer; Noxa, 5'-TCCAGTTGGAGGCTGAGG TT-3' for forward primer, 5'-CACTCGACTTCCAGCTCTGC-3' for reverse primer; Killer5, 5'-CTCCTGCAAATATGGACAGGACTA-3' for forward primer, 5'-TTAGCTCCACTTCACCTGAATCAC-3' for reverse primer.

\section{Apoptosis assays}

hESCs were cultured onto matrigel-coated plates in mTeSR1 medium with $5 \times$ supplement (Invitrogen). hESCs were treated with doxorubicin $(0.2 \mu \mathrm{mol} / \mathrm{L})$, or irradiated with $\gamma$-irradiation (5 Gy), and harvested 8 and $16 \mathrm{~h}$ after treatment. Apoptotic cells were identified by staining with Annexin $\mathrm{V}$ as previously described (Chao et al., 2003).

\section{In vivo ubiquitylation assays}

For in vivo ubiquitylation assays, prior to cell collection, cells were treated with Alln $(25 \mu \mathrm{mol} / \mathrm{L})$, or MG132 $(25 \mu \mathrm{mol} / \mathrm{L})$ for $4 \mathrm{~h}$, and then cells were lysed in modified RIPA buffer ( $1 \%$ Nonidet P- $40,0.1 \%$ sodium dodecyl sulfate [SDS], Tris- $\mathrm{HCl}$ [pH 7.8], $150 \mathrm{mmol} / \mathrm{L} \mathrm{NaCl}$, $1 \mathrm{mmol} / \mathrm{L}$ dithiothreitol, $0.5 \mathrm{mmol} / \mathrm{L}$ EDTA, $25 \mu \mathrm{mol} / \mathrm{L}$ Alln, $25 \mu \mathrm{mol} / \mathrm{L}$ MG132, $5 \mathrm{mmol} / \mathrm{L} \mathrm{N}$-ethylmaleimide, and fresh proteinase inhibitors) with mild sonication. Cell lysates were immunoprecipitated with antip53 antibody (FL393 AC; Santa Cruz Biotechnology, Santa Cruz, $\mathrm{CA}$ ) and analysed by Western blotting using anti-ubiquitin antibody (P4D1; Santa Cruz Biotechnology, Santa Cruz, CA) or anti-p53 antibody (pAb1801; Santa Cruz Biotechnology) (Feng et al., 2005).

Analysis of p53 stability

For in vivo p53 degradation assays, hESCs were incubated with cycloheximide $(100 \mu \mathrm{g} / \mathrm{mL})$. Cells were harvested at 20,40 , and 80 min later, subjected to Western blotting using anti-p53 antibody (pAb1801; Santa Cruz Biotechnology, Santa Cruz, CA). The intensity of bands was measured by densitometry.

\section{Karyotyping analysis}

Karyotyping of the mutant hESC clones was carried out by Cell Line Genetics (Madison, WI).

\section{ACKNOWLEDGMENTS}

This work was supported by grants from California Institute for Regenerative Medicine (RC1-00148) to Y.X. and grants from the National Natural Science Foundation of China (Grant Nos. 81172828 and 81373166 ) to X.F.

\section{COMPLIANCE WITH ETHICS GUIDELINES}

Sun-Ku Chung, Shengyun Zhu, Yang $\mathrm{Xu}$, and Xuemei Fu declare that they have no conflict of interest.

All hESC work in this study has been approved by the Institutional Embryonic Stem Cell Research Oversight Committee (ESCRO). All institutional and national guidelines for the care and use of laboratory animals were followed.

\section{OPEN ACCESS}

This article is distributed under the terms of the Creative Commons Attribution License which permits any use, distribution, and reproduction in any medium, provided the original author(s) and the source are credited.

\section{REFERENCES}

Brooks C, Gu W (2011) The impact of acetylation and deacetylation on the p53 pathway. Protein Cell 2:456-462

Campisi J (2005) Senescent cells, tumor suppression, and organismal aging: good citizens, bad neighbors. Cell 120:513-522

Chao C, Hergenhahn M, Kaeser MD, Wu Z, Saito S, Iggo R, Hollstein M, Appella E, Xu Y (2003) Cell type and promoterspecific roles of Ser18 phosphorylation in regulating p53 responses. J Biol Chem 278:41028-41033

Chao C, Herr D, Chun J, Xu Y (2006) Ser18 and 23 phosphorylation is required for p53-dependent apoptosis and tumor suppression. Embo J 25:2615-2622 Epub 2006 Jun 2611

Cowan CA, Klimanskaya I, McMahon J, Atienza J, Witmyer J, Zucker JP, Wang S, Morton CC, McMahon AP, Powers D et al (2004) Derivation of embryonic stem-cell lines from human blastocysts. N Engl J Med 350:1353-1356 Epub 2004 Mar 1353

Feng L, Lin T, Uranishi H, Gu W, Xu Y (2005) Functional analysis of the roles of posttranslational modifications at the p53 C terminus in regulating p53 stability and activity. Mol Cell Biol 25:5389-5395

Li T, Kon N, Jiang L, Tan M, Ludwig T, Zhao Y, Baer R, Gu W (2012) Tumor suppression in the absence of p53-mediated cell-cycle arrest, apoptosis, and senescence. Cell 149:1269-1283

Lin T, Chao C, Saito S, Mazur SJ, Murphy ME, Appella E, Xu Y (2005) p53 induces differentiation of mouse embryonic stem cells by suppressing Nanog expression. Nat Cell Biol 7:165-171 Epub 2004 Dec 26

Liu D, Ou L, Clemenson GD, Chao C, Lutske ME, Zambetti GP, Gage $\mathrm{FH}, \mathrm{Xu} Y$ (2010) Puma is required for p53-induced depletion of adult stem cells. Nat Cell Biol 12:993-998

Song $\mathrm{H}$, Chung S-K, Xu Y (2010) Modeling disease in human ESCs using an efficient BAC-based homologous recombination system. Cell Stem Cell 6:80-89

Sykes SM, Mellert HS, Holbert MA, Li K, Marmorstein R, Lane WS, McMahon SB (2006) Acetylation of the p53 DNA-binding domain regulates apoptosis induction. Mol Cell 24:841-851

Tang Y, Luo J, Zhang W, Gu W (2006) Tip60-dependent acetylation of p53 modulates the decision between cell-cycle arrest and apoptosis. Mol Cell 24:827-839

Tang Y, Zhao W, Chen Y, Zhao Y, Gu W (2008) Acetylation is indispensable for p53 activation. Cell 133:612-626

Vousden KH, Prives C (2009) Blinded by the light: the growing complexity of p53. Cell 137:413-431

Zhang Z-N, Chung S-K, Xu Z, Xu Y (2014) Oct4 maintains the pluripotency of human embryonic stem cells by inactivating $\mathrm{p} 53$ through Sirt1-mediated deacetylation. Stem Cells 32:157-165 